\title{
Prognostic Factors for Pulmonary Metastasectomy in Hepatocellular Carcinoma
}

\author{
Shuenn-Wen Kuo, ${ }^{1}$ Yih-Leong Chang, ${ }^{2}$ Pei-Ming Huang, ${ }^{1}$ Hsao-Hsun Hsu, ${ }^{1}$ Jin-Shin Chen, \\ Jang-Ming Lee, ${ }^{1}$ Po-Huang Lee, ${ }^{1}$ and Yung-Chie Lee ${ }^{3}$ \\ ${ }^{1}$ Department of Surgery and Traumatology, National Taiwan University Hospital and National Taiwan University College of \\ Medicine, Taipei 100, Taiwan \\ ${ }^{2}$ Department of Surgery and Department of Pathology, National Taiwan University Hospital and National Taiwan University \\ College of Medicine, Taipei 100, Taiwan \\ ${ }^{3}$ Division of Thoracic Surgery, Department of Surgery and Traumatology, National Taiwan University Hospital and National \\ Taiwan University College of Medicine, No. 7 Chung-San S. Rd, Taipei 100, Taiwan
}

\begin{abstract}
Purpose: To identify the prognostic factors for pulmonary metastasectomy (PM-ectomy) in hepatocellular carcinoma (HCC).

Patients and methods: We conducted a retrospective review of patients with pulmonary metastases (PM) from HCC who had undergone curative PM-ectomy at National Taiwan University Hospital between 1990 and 2004. Univariate (log-rank) and multivariate (Cox's model) analyses of survival were used to identify the significant prognostic factors.

Results: In total, 34 patients were eligible for curative PM-ectomy. The overall survival rates (Kaplan-Meier) after PM-ectomy were $65.2 \%$ and $27.5 \%$ at 2 and 5 years, respectively. High alpha-fetoprotein level, positive hepatic resection margin, and short disease-free interval (DFI) were unfavorable factors for overall survival from univariate analysis, however, only DFI $(P=0.028)$ was identified as an independently prognostic factor by multivariate analysis. Bilateral distribution and more PMs were unfavorable factors for PM-free survival from univariate analysis, with only PM number identified as an independent prognostic factor by multivariate analysis $(P=0.017)$.
\end{abstract}

Conclusion: Patients with longer DFIs and fewer PMs can benefit from PM-ectomy in HCC.

Key Words: Hepatocellular carcinoma-Pulmonary metastasis-Metastasectomy.

Pulmonary metastasectomy (PM-ectomy) has been proven effective for miscellaneous neoplasms, such as osteogenic sarcoma, soft tissue sarcoma, renal cell carcinoma, melanoma, and colorectal cancer, with the prognostic factors widely investigated. However, there are few published series of PM-ectomy in HCC, and the sample sizes were small (mostly fewer than ten individuals). ${ }^{1-7}$ Analysis of the available data

Received August 5, 2006; accepted August 8, 2006; published online December 6, 2006.

Address correspondence and reprint requests to: Yung-Chie Lee, Division of Thoracic Surgery, Department of Surgery, National Taiwan University Hospital, No. 7 Chung-San S. Rd, Taipei 100, Taiwan; E-mail: wuj@ha.mc.ntu.edu.tw

Published by Springer Science+Business Media, Inc. (c) 2006 The Society of Surgical Oncology, Inc. only permits the conclusion that PM-ectomy for HCC offers a chance for disease control and longterm survival. However, there is no reliable indicator to facilitate selection of surgical candidates who may benefit from this procedure. Thus, the purpose of this article is to present our experience with PM-ectomy for HCC and to examine the clinical factors that may be important for predicting outcome.

\section{PATIENTS AND METHODS}

\section{Patient Population}

We retrospectively assessed patients who underwent a PM-ectomy for hepatocellular carcinoma (HCC) at 
National Taiwan University Hospital between 1990 and 2004. The observation period ended on January 31, 2006. Thirty-four patients (30 males and 4 females) were deemed eligible for curative PM-ectomy based on the following criteria: (1) primary tumor locally controlled; (2) extrathoracic metastases absent; (3) radical PM-ectomy possible; and (4) sufficient pulmonary function preserved post-PM-ectomy. Thirteen patients developed hepatic recurrence before the appearance of PM with: six undergoing re-resection of the hepatic tumor; transcatheter arterial chemoembolization used for effective control of their hepatic tumors in six; and, the remaining individuals underwent re-resection for the first recurrence and transcatheter arterial chemoembolization for the second.

\section{Surgical Treatment and Follow-up}

The PM-ectomy was performed under general anesthesia with controlled ventilatory support in all cases. The PM was approached through an open thoracotomy, sternotomy or thoracoscopy. The metastatic lesion was resected using a pulmonary wedge resection or lobectomy. Dissection of the mediastinal lymph node was not undertaken routinely unless enlarged lymph nodes were seen on preoperative computed tomography (CT). After the PM-ectomy, patients were followed up regularly at intervals of 1-3 months by means of physical examination, chest radiography, serum alpha-fetoprotein and ultrasonography of the liver remnant. CT of the lung and abdomen were performed if any abnormality was noted.

\section{Variables and Definitions}

The 16 clinicopathological variables were analyzed and categorized into three groups: (1) host factors: age at PM-ectomy, gender, presence of liver cirrhosis and hepatitis virus; (2) primary tumor factors: (a) initial alpha-fetoprotein level, time interval between primary hepatic surgery and appearance of recurrence at any site (disease-free interval: DFI), (b) vascular invasion, satellite lesion, size, histological grading (Edmondson and Stainer's system) and resection margin of the hepatic tumor; (3) PM factors: laterality of the PM distribution, number of pathologically confirmed PMs, size of the largest PM, resection extent (lobectomy versus wedge resection) and approach method (open versus thoracoscopic; Table 1). Thoracotomy and sternotomy, which allowed bimanual palpation of the whole lung, are classified as open methods. Surgical outcome was evaluated from the overall and PM-free survivals, which were calculated from the dates of PMectomy to last known follow-up/death, and, to the appearance of PM or last known follow-up/death, respectively.

\section{Statistical Methods}

The Kaplan-Meier method was used to estimate the survival probabilities. Deaths from causes other than tumor were classified as censored. The prognostic influence of variables on survival was analyzed using the log-rank test for univariate analysis and the Cox proportional hazards model for multivariate analysis. Variables found to be significantly prognostic factors in the univariate analysis were tested using the multivariate analog. Data were considered significant when the Wald statistic $P<0.05$. SPSS statistical software was used for all statistical analysis.

\section{RESULTS}

\section{Patients}

The mean age of the 30 males and 4 females was 56 years (range, 28-81, median 53.5). Twenty-three patients had been diagnosed with chronic hepatitis B, two chronic hepatitis $\mathrm{C}$, and three dual infections. The range of alpha-fetoprotein serum level before hepatic resection was $5-441,000 \mathrm{ng} / \mathrm{ml}$. The mean size of the primary HCC was $8.3 \mathrm{~cm}$ (range 2-15, median 9); mean DFI was 15.7 months (range 2-65, median 11.5); mean size of the largest PM was $2.9 \mathrm{~cm}$ (range 1-10, median 2); mean PM number was 2.4 (range 1-21, median 1); and mean post-PM-ectomy followup interval was 30 months (range 2-115, median 23.5). The overall survival rates were $65.2 \%$ and $27.5 \%$ at 2 and 5 years, respectively. The median survival was 56 months.

\section{Pattern of Failure}

During the follow-up period, 19 of the 34 patients expired (crude mortality rate 55.9\%), 15 due to HCC progression $(n=15)$ and 4 from other causes [gastrointestinal bleeding $(n=2)$, sepsis $(n=1)$, and postoperative ARDS $(n=1)$ ]. Mean survival from PM-ectomy to death was 20 months. At the end of follow-up, 10 of the 15 survivors remained diseasefree (four of them had undergone a second PM-ectomy), with the remaining five individuals experiencing recurrence. At the time of writing, one patient had survived disease-free for 115 months after PM-ectomy. 
TABLE 1. Actuarial survival rates according to various clinical factors

\begin{tabular}{|c|c|c|c|c|c|c|c|}
\hline \multirow[b]{2}{*}{ Factor } & \multirow[b]{2}{*}{ No. of patients } & \multicolumn{3}{|c|}{ Overall survival } & \multicolumn{3}{|c|}{ PM- free survival } \\
\hline & & 2 years $(\%)$ & 5 years $(\%)$ & $P$ value & 1 year $(\%)$ & 5 years $(\%)$ & $P$ value \\
\hline \multicolumn{8}{|l|}{ Host factors } \\
\hline \multicolumn{8}{|l|}{ Age (years) } \\
\hline$<55$ & 19 & 60.2 & 52.7 & \multirow[t]{2}{*}{0.939} & 59.4 & 44.5 & \multirow[t]{2}{*}{0.844} \\
\hline$\geq 55$ & 15 & 62.9 & 50.3 & & 57.4 & 43.1 & \\
\hline \multicolumn{8}{|l|}{ Gender } \\
\hline Male & 30 & 55.8 & 45.1 & \multirow[t]{2}{*}{0.246} & 56.7 & 45.4 & \multirow[t]{2}{*}{0.703} \\
\hline Female & 4 & 100 & $-*$ & & 75 & $-*$ & \\
\hline \multicolumn{8}{|l|}{ Cirrhosis } \\
\hline- & 18 & 76 & 57.9 & \multirow{2}{*}{0.327} & 58.2 & 49.9 & \multirow{2}{*}{0.617} \\
\hline+ & 16 & 45 & 22.5 & & 59.8 & 35.9 & \\
\hline \multicolumn{8}{|l|}{ Viral hepatitis } \\
\hline- & 6 & 62.5 & $-*$ & \multirow[t]{2}{*}{0.574} & 50 & $-*$ & \multirow[t]{2}{*}{0.967} \\
\hline+ & 28 & 60.5 & 24.4 & & 61.2 & 42.8 & \\
\hline Primary tumor $f$ & & & & & & & \\
\hline Alpha-fetoprot & & & & & & & \\
\hline$<100 \mathrm{ng} / \mathrm{ml}$ & 15 & 83.9 & 69.9 & 0.029 & 70 & 52.5 & 0.329 \\
\hline$\geq 100 \mathrm{ng} / \mathrm{ml}$ & 19 & 44.7 & 19.1 & & 50.2 & 37.7 & \\
\hline DFI (months) & & & & & & & \\
\hline$<12$ & 17 & 38.5 & 28.9 & 0.011 & 51 & 38.2 & 0.385 \\
\hline$\geq 12$ & 17 & 86.7 & 78 & & 66.6 & 49.9 & \\
\hline First recurrence s & & & & & & & \\
\hline Liver & 13 & 41.7 & 27.8 & 0.202 & 49.3 & 37 & 0.322 \\
\hline Lung & 21 & 75 & 67.5 & & 63.6 & 47.7 & \\
\hline Vascular invasion & & & & & & & \\
\hline- & 13 & 81.8 & 65.5 & 0.336 & 64.6 & 38.8 & 0.937 \\
\hline+ & 21 & 51 & 43.7 & & 54.3 & 46.5 & \\
\hline Satellite lesion & & & & & & & \\
\hline- & 20 & 62.7 & 55.8 & 0.76 & 41.3 & 34.4 & 0.091 \\
\hline+ & 14 & 58.6 & 23.4 & & 85.1 & 56.7 & \\
\hline Tumor size & & & & & & & \\
\hline$<10 \mathrm{~cm}$ & 20 & 52.1 & 41.7 & 0.217 & 56.1 & 40.1 & 0.618 \\
\hline$\geq 10 \mathrm{~cm}$ & 14 & 75.2 & 32.9 & & 61.9 & 49.5 & \\
\hline Tumor grading & & & & & & & \\
\hline Low (I-II) & 10 & 88.9 & 55.6 & 0.269 & 66.7 & 44.4 & 0.802 \\
\hline High (III-IV) & 24 & 49 & 24.5 & & 55.4 & 46.2 & \\
\hline Resection margin & & & & & & & \\
\hline$<1 \mathrm{~cm}$ & 30 & 68.1 & 28.7 & 0.04 & 61.2 & 45.9 & 0.49 \\
\hline$\geq 1 \mathrm{~cm}$ & 4 & 0 & 0 & & 33.3 & $-*$ & \\
\hline PM factors & & & & & & & \\
\hline Laterality & & & & & & & \\
\hline Unilateral & 25 & 58.8 & 25.7 & 0.865 & 68.5 & 53.3 & 0.028 \\
\hline Bilateral & 9 & 66.7 & 55.6 & & 33.3 & 22.2 & \\
\hline PM number & & & & & & & \\
\hline$<3$ & 23 & 65.6 & 29.2 & 0.48 & 80 & 64 & $<0.001$ \\
\hline$\geq 3$ & 11 & 51.9 & $-*$ & & 18.2 & $-*$ & \\
\hline Largest $P M$ size & & & & & & & \\
\hline$<3 \mathrm{~cm}$ & 18 & 62.7 & 45.7 & 0.78 & 53.3 & 53.3 & 0.895 \\
\hline$\geq 3 \mathrm{~cm}$ & 16 & 58.7 & 29.3 & & 66.3 & 37.9 & \\
\hline Resection extent & & & & & & & \\
\hline Lobectomy & 11 & 70 & 35 & 0.467 & 77.1 & 46.3 & 0.283 \\
\hline Wedge & 23 & 56.7 & 44.7 & & 49.9 & 42.8 & \\
\hline Approach metho & & & & & & & \\
\hline Open $^{\dagger}$ & 19 & 58.2 & 29.1 & 0.852 & 64.3 & 36.7 & 0.894 \\
\hline Thoracoscopic & 15 & 64 & 45.7 & & 52.5 & 52.5 & \\
\hline
\end{tabular}

Abbreviations: DFI, disease-free interval; PM, Pulmonary metastases.

* All patients in this group were not followed up to 5 years.

$\dagger$ Thoracotomy or sternotomy.

\section{Prognostic Factors}

Potential prognostic factors for overall and PM-free survivals were tested by univariate analysis
(Table 1). Patients with higher alpha-fetoprotein level, closer hepatic resection margin or shorter DFI had inferior outcomes in terms of the overall survival (Fig. 1). Unilateral lung involvement and lower PM 


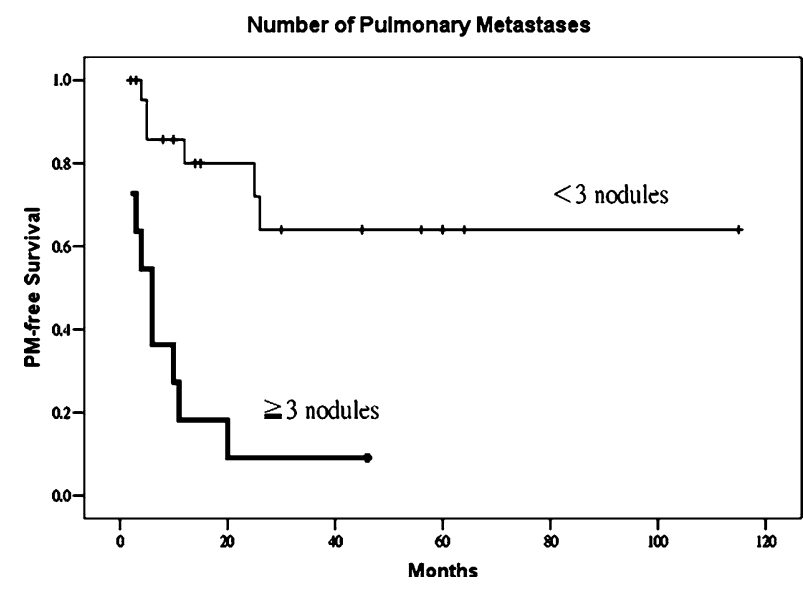

FIG. 1. Overall survival in relation to disease-free interval (DFI). Longer DFI was associated with significantly longer survival $(P=0.011)$.

number were favorable factors in terms of PM-free survival (Fig. 2). From multivariate analysis, only DFI and PM number were found to be independent predictors of overall survival and PM-free survival, respectively (Table 2).

\section{DISCUSSION}

HCC has been the major cause of cancer death in Taiwan and East Asia for many years because of the high prevalence of viral hepatitis in this region. Surgical resection is the optimal treatment for primary and locally recurrent $\mathrm{HCC} .{ }^{8}$ Once distant metastasis occurs, however, these patients are deemed terminal because an effective systemic therapy is not available. Okusaka et al. reported a median survival of 4.6 months for a sample of 34 patients with metastatic $\mathrm{HCC}$ who received chemotherapy, with a $20.3 \%$ 1-year survival rate. ${ }^{9}$ Lo et al. performed surgical resection for 12 patients with metastatic HCC, who had longer survivals compared with another 24 who were treated nonoperatively (52\% versus $21 \%$ and $26 \%$ versus $0 \%$ at 2 and 5 years postprimary hepatectomy, respectively). ${ }^{1}$ Pulmonary involvement was the most prevalent in terms of possible metastatic sites for $\mathrm{HCC},{ }^{4,9-11}$ with PM-ectomy preferred by many authors for intent to prolong survival. ${ }^{1-7}$ Postprocedure survival rates for HCC were analyzed in two reports. Lam et al. reported a $10 \%$ 5-year survival rate $^{4}$ and Nakajima et al. revealed a $23.8 \%$ 3 -year survival rate. ${ }^{2}$ In the present report, the survival rate after PM-ectomy was $65.2 \%$ at 2 years and $27.5 \%$ at 5 years. According to data mentioned here, operative groups appear to have better survival

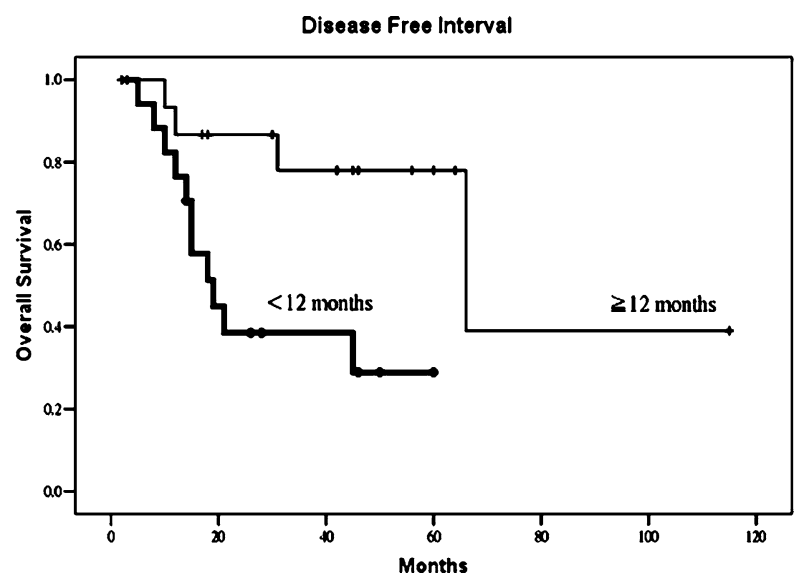

FIG. 2. Pulmonary metastases (PM)-free survival with relation to PM number. Larger PM number was associated with significantly shorter PM-free survival $(P<0.001)$.

TABLE 2. Multivariate analysis

\begin{tabular}{lllll}
\hline & Variable & $\begin{array}{l}\text { Hazard } \\
\text { ratio }\end{array}$ & $\begin{array}{l}\text { 95\% confidence } \\
\text { interval }\end{array}$ & $P$ value \\
\hline $\begin{array}{l}\text { Overall survival } \\
\text { PM-free survival }\end{array}$ & PFI & 0.85 & $0.73-0.9$ & 0.028 \\
\hline $\begin{array}{c}\text { Abbreviations: } \\
\text { metastases. }\end{array}$ & DFI, disease-free & interval; PM, Pulmonary \\
$*$ Continuous variables; the unit of DFI is months. &
\end{tabular}

results than historic analogs treated nonoperatively. ${ }^{1,9}$ However, selection bias is inevitable because patients with good clinical condition are often those deemed eligible for surgical treatment.

The survival rates after HCC PM-ectomy were low when compared with the analogous procedures used for other tumors. The range of 5-year survival rates post-PM-ectomy in several large patient series is $35-48 \%$ for miscellaneous neoplasms. ${ }^{12-14}$ The bleaker prognosis for HCC may be attributable to its naturally malignant characteristics and that should serve as a reminder of the importance of prognostic factors when selecting candidates for PM-ectomy. Widely investigated in the literature, the prognostic factors are DFI, PM number and size and complete PM resection. The results of prognostic analysis for HCC PM-ectomy have only been reported for one sample of 20 patients; however, no significant factor affecting overall survival was identified. ${ }^{2}$

In the present series, the host and PM factors did not significantly influence overall survival. Of the primary tumor factors, high alpha-fetoprotein level (?100), close resection margin $(<1 \mathrm{~cm})$ and short DFI ( $<12$ months) were associated with a significant decrease in the overall survival from univariate 
analysis. Peng et al. also indicated that high alphafetoprotein level was a significantly unfavorable prognostic factor in patients undergoing curative hepatectomy of primary HCC. ${ }^{15}$ Nakajima et al. demonstrated that positive resection margin decreased the disease-free survival but did not affect overall survival. ${ }^{2}$ In our study, DFI was an independently prognostic indicator from multivariate analysis (Table 2), however, the significance demonstrated by Nakajima et al. was not confirmed. ${ }^{2}$ These researchers defined DFI as the interval between primary liver surgery and PM diagnosis, however, we suggest that the interval to cancer recurrence (not restricted to lung) can better reflect the aggressiveness of the primary tumor. The shorter DFI is because of the more aggressive biology of the primary tumor, and ultimately determines the poorer overall survival. ${ }^{12,16-20}$ In the present study, DFI remained a significant factor in Cox's model, where DFI (in months) is taken as a continuous variable, avoiding the bias inherent in adoption of a single, occasionally arbitrary, cutoff value in univariate analysis.

Should the patient with unfavorable factors not be considered as a candidate for PM-ectomy? What is the minimum DFI for HCC PM-ectomy? These questions cannot be answered based on the currently available information. Our data show that the overall survival for patients with shorter DFI ( $<12$ months) was still superior to that of analogs treated nonoperatively. ${ }^{1,9}$ However, we cannot arbitrarily conclude that all patients with PM from HCC should undergo PM-ectomy. Although selection bias and small sample size are the limitations of the present study, conducting a prospective, randomized trial is problematic because of the small number of candidates available for HCC PM-ectomy. Case-match study may provide an alternative investigative method to resolve these issues.

In our study, host and primary tumor factors did not significantly influence PM-free survival. Of the PM factors, bilateral PM distribution and larger PM number were significantly unfavorable prognostic factors from univariate analysis. Further, only PM number was an independent prognostic indicator by multivariate analysis (Table 2). Larger PM number may suggest that the biology of the tumor has already predisposed the patient to a more disseminated course. Additionally, early recurrence may indicate the growth of originally micrometastatic lesions, which cannot be detected by CT scan, or by bimanual lung palpation during operation. Verazin et al. also reported that patients with fewer metastases have a longer disease-free survival. ${ }^{21}$ Thus, careful follow-up with more frequent chest X-ray examination is mandatory for patients at high risk of PM recurrence, especially those with larger PM numbers.

The efficacy of metastasectomy for multiple lesions has generated both controversy and skepticism. Analysis of our data reveals no association between PM number and overall survival, however, there was a relationship between PM number and PM-free survival. Similar findings were also reported for patient samples with PMs of colorectal cancer. ${ }^{22,23}$ However, a prognostic analysis of 5,206 cases undergoing PM-ectomy for miscellaneous tumor types has demonstrated that single PM is a favorable prognostic factor compared with multiple PMs. ${ }^{12}$ This discrepancy made PM number controversial in patient selection for PM-ectomy. Investigating patients with PM from HCC, Lam et al. used solitary PM for their operative criterion, ${ }^{4}$ but other researchers did not exclude multiple PMs. ${ }^{1,2,5-7}$ More studies involving larger samples of patients with a single cancer are needed to settle this controversy. As discussed here, however, the study of PM from HCC remains somewhat problematic.

The resection extent of PM-ectomy has become less and less. Currently, most surgeons appear to consider wedge resection the procedure of choice, with emphasis on preservation of a normal pulmonary parenchyma. ${ }^{24}$ In the present study, no significant relationship was demonstrated between the extent of PM-ectomy and survival, confirming the findings of Mineo et al. for the procedure for miscellaneous tumor types. ${ }^{25}$ Further, as wedge resection can be used for complete removal of most PMs given their typically peripheral location, we suggest that this is the optimal procedure for PM-ectomy in HCC.

The surgical approach for PM-ectomy is also controversial. Some authors prefer the thoracoscopic technique, which is associated with reduced morbidity and shorter admission. Additionally, analysis of our data did not reveal any difference between the open and thoracoscopic approaches with respect to overall and PM-free survivals. However, the opinion against thoracoscopic approach is largely based on the inability to perform bimanual palpation of the whole lung to detect subclinical lesions missed with traditional CT scan. ${ }^{24}$ It appears likely that the probability of this false negative finding will decrease as CT imaging technology and related techniques continue their rapid and seemingly inexorable advance. The multislice spiral CT improves nodule detection because the entire lung can be scanned at high resolution during a single breath hold without an intersection gap. Nevertheless, a prospective, 
randomized comparison trial of the thoracoscopic and open surgery using spiral CT scan to identify lesions preoperatively is needed to settle this debate conclusively.

Repeat PM-ectomy for recurrent disease can be performed with low mortality and improved survival. ${ }^{24}$ Five of our 26 patients suffering PM recurrence were eligible for a second PM-ectomy; four were alive at the time of writing without evidence of disease recurrence. Jacklitsch et al. analyzed outcome for patients undergoing multiple PM-ectomies and concluded that multiple attempts to reestablish intrathoracic control of the metastatic disease were justified in carefully selected patients. ${ }^{26}$

This study confirms the proposition that longer DFI is associated with greater benefit from PM-ectomy in HCC. Larger number of PMs is associated with higher risk of PM recurrence and more frequent follow-up is mandatory for patients at risk of disease recurrence. However, short DFI and large PM number should not constitute absolute contraindications to surgical treatment. Further studies are needed to more clearly delineate the relevant prognostic factors, which will facilitate superior definition of the surgical indications for patients with PM from HCC.

\section{REFERENCES}

1. Lo CM, Lai EC, Fan ST, et al. Resection for extrahepatic recurrence of hepatocellular carcinoma. Br J Surg 1994; 81(7):1019-1021.

2. Nakajima J, Tanaka M, Matsumoto J, et al. Appraisal of surgical treatment for pulmonary metastasis from hepatocellular carcinoma. World J Surg 2005; 29(6):715-718.

3. Utsunomiya T, Shimada M, Shirabe K, et al. Clinicopathological characteristics of patients with extrahepatic recurrence following a hepatectomy for hepatocellular carcinoma. Hepato-Gastroenterology 2001; 48(40):1088-1093.

4. Lam CM, Lo CM, Yuen WK, et al. Prolonged survival in selected patients following surgical resection for pulmonary metastasis from hepatocellular carcinoma. Br J Surg 1998; 85(9):1198-1200.

5. O'Suilleabhain CB, Poon RT, Lau CW, Fan ST. Repeated resections of extrahepatic metastases after hepatic resection: an aggressive approach to hepatocellular carcinoma. Hepatogastroenterology 2004; 51(57):82582-9.

6. Gwak GY, Jung JO, Sung SW, Lee HS. Long-term survival after pulmonary metastatectomy of hepatocellular carcinoma; treatment outcome or natural history? Hepatogastroenterology 2004; 51(59):1428-1433.
7. Chen YJ, Hsu HS, Hsieh CC, et al. Pulmonary metastasectomy for hepatocellular carcinoma. J Chin Med Assoc 2004; 67(12):621-624.

8. Carr BI. Hepatocellular carcinoma: current management and future trends. Gastroenterology 2004; 127(5 Suppl 1):S218-S224.

9. Okusaka T, Okada S, Ishii H, et al. Prognosis of hepatocellular carcinoma patients with extrahepatic metastases. Hepatogastroenterology 1997; 44(13):251-257.

10. Lee YT, Geer DA. Primary liver cancer: pattern of metastasis. $J$ Surg Oncol 1987; 36(1):26-31.

11. Tsai GL, Liu JD, Siauw CP, Chen PH. Thoracic roentgenologic manifestations in primary carcinoma of the liver. Chest 1984; 86(3):430-434.

12. Pastorino U, Buyse M, Friedel G. Long-term results of lung metastasectomy: prognostic analyses based on 5206 cases. The International Registry of Lung Metastases. $J$ Thorac Cardiovasc Surg 1997; 113(1):37-49.

13. Mountain CF, McMurtrey MJ, Hermes KE. Surgery for pulmonary metastasis: a 20-year experience. Ann Thorac Surg 1984; 38(4):323-330.

14. Robert JH, Ambrogi V, Mermillod B, et al. Factors influencing long-term survival after lung metastasectomy. Ann Thorac Surg 1997; 63(3):777-784.

15. Peng SY, Chen WJ, Lai PL, et al. High alpha-fetoprotein level correlates with high stage, early recurrence and poor prognosis of hepatocellular carcinoma: significance of hepatitis virus infection, age, p53 and beta-catenin mutations. Int $J$ Cancer 2004; 112(1):44-50.

16. Frost DB. Pulmonary metastasectomy for soft tissue sarcomas: is it justified?. J Surg Oncol 1995; 59(2):110-115.

17. Poon RT, Fan ST, Ng IO, et al. Different risk factors and prognosis for early and late intrahepatic recurrence after resection of hepatocellular carcinoma. Cancer 2000; 89(3):500-507.

18. Marincola FM, Mark JB. Selection factors resulting in improved survival after surgical resection of tumors metastatic to the lungs. Arch Surg 1990; 125(10):1387-1392; discussion 1392-1393.

19. van Geel AN, Pastorino U, Jauch KW, et al. Surgical treatment of lung metastases: The European Organization for Research and Treatment of Cancer-Soft Tissue and Bone Sarcoma Group study of 255 patients. Cancer 1996; 77(4):675-682.

20. Shimada M, Takenaka K, Gion T, et al. Prognosis of recurrent hepatocellular carcinoma: a 10-year surgical experience in Japan. Gastroenterology 1996; 111(3):720-726.

21. Verazin GT, Warneke JA, Driscoll DL, et al. Resection of lung metastases from soft-tissue sarcomas. A multivariate analysis. Arch Surg 1992; 127(12):1407-1411.

22. Rizk NP, Downey RJ. Resection of pulmonary metastases from colorectal cancer. [Review] [42 refs]. Semin Thorac Cardiovasc Surg 2002; 14(1):29-34.

23. McCormack PM, Ginsberg RJ. Current management of colorectal metastases to lung. Chest Surg Clin N Am 1998; 8(1):119-126.

24. Rusch VW.. Pulmonary metastasectomy. Current indications. Chest 1995; 107(6 Suppl):322S-331S.

25. Mineo TC, Ambrogi V, Tonini G, Nofroni I. Pulmonary metastasectomy: might the type of resection affect survival? J Surg Oncol 2001; 76(1):47-52.

26. Jaklitsch MT, Mery CM, Lukanich JM, et al. Sequential thoracic metastasectomy prolongs survival by re-establishing local control within the chest. J Thorac Cardiovasc Surg 2001; 121(4):657-667. 